\title{
Imperfect Radio Over Fibre Aided Distributed Antennas with Fractional Frequency Reuse
}

\author{
Xinyi Xu, Rong Zhang and Lajos Hanzo \\ School of ECS., Univ. of Southampton, SO17 1BJ, UK. \\ Tel: +44-23-80-593 125, Fax: +44-23-80-593045 \\ Email: 1h@ecs.soton.ac.uk, http://www-mobile.ecs.soton.ac.uk
}

\begin{abstract}
The achievable throughput of the entire cellular area is investigated, when employing fractional frequency reuse techniques in conjunction with realistically modelled imperfect optical fibre aided distributed antenna systems (DAS) operating in a multicell scenario. Given a fixed total transmit power, a substantial improvement of the cell-edge area's throughput can be achieved without reducing the cell-centre's throughput. The distributed antennas are connected by realistic lossy optical fibre associated with a pulse-broadening parameter of $\beta \leq 0.1$. Quantitatively, the entire cell-edge throughput is better than $\eta=$ $3 \mathrm{bits} / \mathrm{s} / \mathrm{Hz}$, potentially reaching $4 \mathrm{bits} / \mathrm{s} / \mathrm{Hz} \leq \eta \leq 5 \mathrm{bits} / \mathrm{s} / \mathrm{Hz}$ in the best illuminated areas. The geographic throughput distribution is characterized with the aid of throughput contours.
\end{abstract}

\section{INTRODUCTION}

The classic Unity Frequency Reuse (UFR) pattern typically has a low throughput at the cell-edge. As a remedy, the Fractional Frequency Reuse (FFR) [1] philosophy may be invoked, which is capable of improving the cell-edge Signalto-Interference-plus-Noise-Ratio (SINR). Similarly, DASs are also capable of attaining an improved coverage, hence increasing the attainable throughput of the cell-edge area and reducing the total transmit power dissipation [2]. In contrast to employing a vulnerable wireless backhaul for connecting the central Base Station (BS) and the distributed Remote Antennas (RAs), the Radio over Fibre (RoF) [3] transmission technique is eminently applicable to construct the BS to RA links by exploiting the benefits of optical fibre based backhauls, although naturally, this increases the system's cost. Hence the capacity of the resultant RoF aided DAS is essentially only limited by the 'last-mile' wireless link.

More explicitly, the FFR technique improves the cell-edge throughput by orthogonally partitioning the entire frequency band to ensure that the co-channel interference at the cell-edge is reduced at the cost of a reduced area-spectral-efficiency. By contrast, the DAS improves the cell-edge throughput by placing the antennas more close to the cell-edge terminals, hence the pathloss is reduced. Thus, it becomes reasonable to combine the benefits of these two techniques in order to resolve, whether the drawbacks of the FFR technique may be compensated by the throughput enhancement of DASs.

Acknowledgements: The financial support of the EPSRC under the auspice of the UK-India Advanced Technology Centre in Wireless Communications as well as that of the EU under the auspices of optimist project is gratefully acknowledged.
Against this background, in this paper we investigate the throughput across the entire cellular coverage area, when employing the FFR technique beneficially combined with realistically modelled imperfect optical fibre aided DAS operating in a multicell scenario. We will demonstrate that

1) The combination of the FFR technique and DAS may not always lead to a combined throughput enhancement, when the pulse-broadening effects of realistic RoF links are also taken into account;

2) Employing different configurations of the RAs in DASs results into different geographic throughput distributions, suggesting that the positioning of the RAs is dependent on the systems geographic user-distribution.

Our paper is organised as follows. In Section II we introduce the multicell topology considered and outline the received signal model. Our link level results recorded for a range of modulation and coding schemes are presented in Section II. This is followed by our performance evaluations in Section III. Finally, we conclude our discourse in Section IV.

\section{OpticAl Fibre Aided DAS with FFR}

\section{A. Multicell System Topology}

The multicell scenario considered in our paper is illustrated in Fig. 1, which consists of seven adjacent hexagonal cells, each having a radius of $R$. The distance between any two adjacent BSs is $D=\sqrt{3} R$, e.g. $\overline{B_{2} B_{3}}=\sqrt{3} R$, as shown in Fig. 1. Based on the FFR regime of this system, the total available bandwidth is divided into the cell-centre frequency band $F_{c}$ and cell-edge frequency band $F_{e}$, where we have $F_{c} \cap F_{e}=\oslash$. Furthermore, the cell-edge frequency band $F_{e}$ is divided into three orthogonal frequency bands, i.e. $F_{1}, F_{2}$ and $F_{3}$, where each frequency band is used exclusively at the cell-edge of one of the three adjacent cells, as seen in Fig. 1.

We assume symmetry, so that every cell has the same system configurations and focus our discussions on cell $B_{0}$ without any loss of generality. The cell-centre area has a radius of $r$. When DASs are employed, the $i$ th RA is described using its polar coordinates denoted by $\left[\theta_{R i}, L_{R i}\right]=[2 \pi(i-$ $1) / K, L], i \in[1, K]$, where $K$ stands for the total number of RAs. Furthermore, the destination Mobile Station (MS) roaming at any point $Z$ within cell $B_{0}$ is described by its polar coordinates of $\left[\theta_{Z}, L_{Z}\right]$. Likewise, the BSs are described by their polar coordinates $\left[\theta_{B j}, L_{B j}\right]=[2 \pi(j-1) / 6, \sqrt{3} R], j \in$ $[1,6]$, while $B_{0}$ assumed to be located at the origin. 


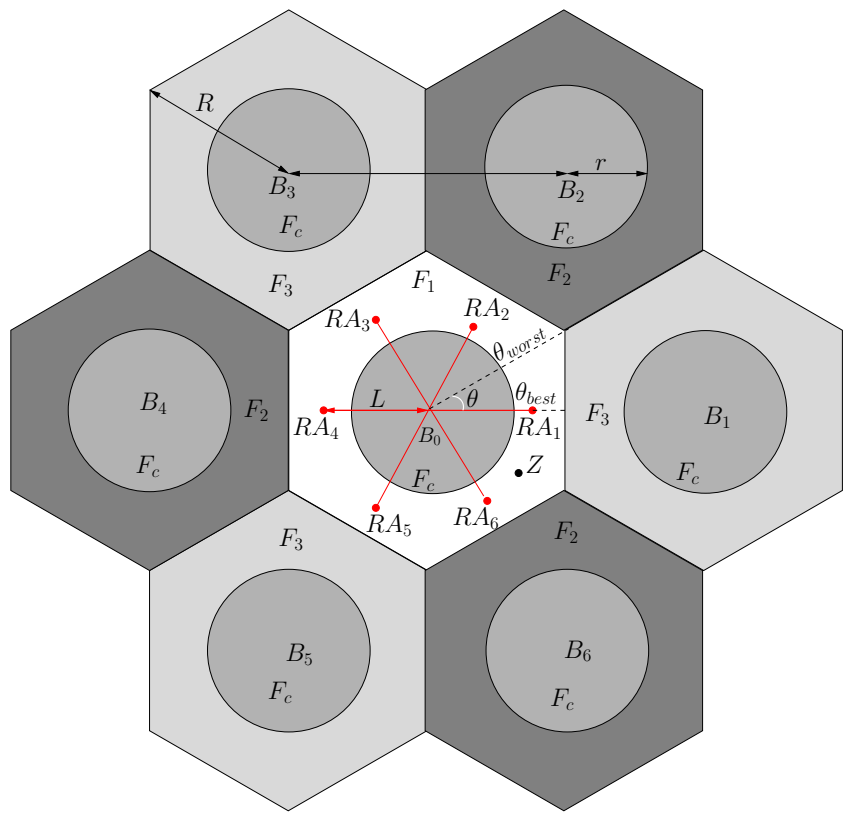

Fig. 1. The toplogy of seven cells relying on a FFR and DAS arrangement, where $K=6$ RAs are employed.

\section{B. Received Signal-to-Interference-plus-Noise-Ratio}

Focusing on the cell $B_{0}$ of Fig. 1, when DASs are employed, a total transmit power constraint $P$ should be imposed, hence we have $P_{B}+K P_{R}=P$, where $P_{B}$ represents the transmit power at the BS and $P_{R}$ denotes the transmit power of each RA, which is assumed to be the same for all RAs.

1) Cell-Centre Area: Considering the cell-centre first, the interference at any MS located at point $Z=\left[\theta_{Z}, L_{Z}\right], L_{Z}<r$ is imposed by the direct wireless links of all tier-one BSs $\left\{B_{1}, \ldots, B_{6}\right\}$, which we refer to as the Inter-BS-Interference (IBI). Hence, the received signal may be written as

$$
y_{c}=\sqrt{P_{B}} \psi_{B 0} h_{B 0} x_{0}+\underbrace{\sum_{j=1}^{6} \sqrt{P_{B}} \psi_{B j} h_{B j} x_{j}}_{I B I}+n,
$$

where $\psi_{B i}, h_{B i}, i \in[0,6]$ and $n \sim \mathcal{C N}\left(0, N_{0}\right)$ represent the large-scale signal attenuation, small-scale fast Rayleigh fading and the complex-valued AWGN, respectively. More explicitly, we jointly consider both the pathloss and the lognormal shadowing component, which is formulated as $\psi_{B i}=$ $\left[\rho 10^{\xi\left(\sigma_{s}\right) / 10}\right]^{1 / 2}, i \in[0,6]$, where $\rho$ denotes the pathloss that obeys a predefined pathloss model [4], while $\xi$ denotes a real valued Gaussian random variable having a standard deviation of $\sigma_{s}=8 \mathrm{~dB}$ [4]. Hence, $G_{B j}=\psi_{B j}\left|h_{B j}\right|$ denotes the equivalent channel gain, while the SINR of any of the MSs near the cell-centre is given by:

$$
\gamma_{c}=\frac{G_{B 0}^{2} P_{B}}{N_{0}+\sum_{j=1}^{6} G_{B j}^{2} P_{B}} .
$$

2) Cell-Edge Area: On the other hand, when considering the cell-edge area, the interference is predominantly imposed by the RAs using the same frequency band within the same cell, which we refer to as the Inter-RA-Interference (IRI).
We assume that the links spanning from the $\mathrm{BS} B_{0}$ to the RAs are constituted by an imperfect optical fibre, while the last-mile connection from the RAs to the MS relies on the wireless link. Hence, the signal received at the $i$ th RA after passing through the 'lossy' optical fibre may be written as $y_{i}=A_{f}\left(A_{p} x_{i}+I_{p}\right)+n_{f}$. More explicitly, we have $A_{f}=$ $\exp (-\alpha L / 2)$ and $n_{f} \sim \mathcal{C} \mathcal{N}\left(0, \sigma_{f}\right)$, where the lossy optical fibre's attenuation coefficient is $\alpha$ and the complex-valued Additive White Gaussian Noise (AWGN) is $n_{f}$. When the wavelength of light is $\lambda=1.55 \mu \mathrm{m}$, a typical value for the attenuation coefficient is $\alpha=0.2 \mathrm{~dB} / \mathrm{km}$ [5]. Furthermore, the so-called pulse-broadening effect of the optical fibre is also taken into account, where the pulse propagated over the fibre is subject to both peak-power reduction and to Inter-PulseInterference (IPI). The peak-power reduction is given by a factor of [6]: $A_{p}=T_{0} /\left(T_{0}^{2}-i \kappa^{2} L^{2}\right)^{1 / 2}$, where $T_{0}=10$ ps stands for the Half-Width-Half-Maximum (HWHM) of the initial Gaussian signalling pulse, while $\kappa=20 \mathrm{ps}^{2} / \mathrm{km}$ is a constant. The IPI is accumulated as a result of the pulse broadening, which may be modelled as a linear function of the optical fibre's length $L$, yielding $I_{p}=\beta L$, with $\beta$ being a fibre-length-dependent constant in the range of 0 and 1 .

The noise-contaminated, faded signal $y_{i}$ at the wireless channel's output is normalised by a power-scaling factor and then forwarded through the wireless channel to the destination, namely to the MS roaming in the cell-edge area, where the power-scaling factor $\chi$ is employed in order to maintain a constant transmit power at the RAs. As a result, the signal received by the MS at point $Z=\left[\theta_{Z}, L_{Z}\right], r<L_{Z}<R$ served by the $i$ th RA may be written as

$$
y_{e}=\sqrt{P_{R}} \psi_{R i} h_{R i} \chi y_{i}+\underbrace{\sum_{k=1, k \neq i}^{K} \sqrt{P_{R}} \psi_{R k} h_{R k} \chi y_{k}}_{I R I}+n .
$$

Let $A=A_{f} A_{p}$ and $N_{f}=\left(A_{f} I_{p}\right)^{2}+2 \sigma_{f}^{2}$ denote the equivalent optical fibre attenuation factor and equivalent optical fibre noise variance, respectively. Then, the SINR at any MS roaming in the cell-edge area is given by:

$$
\gamma_{e}=\frac{G_{R i}^{2} A^{2} P_{R}}{N_{0}+\sum_{i=1}^{K} G_{R i}^{2} N_{f} P_{R}+\sum_{k=1, k \neq i}^{K} G_{R k}^{2} A^{2} P_{R}},
$$

where $G_{R i}=\psi_{R i}\left|h_{R i}\right| \chi$ denotes the equivalent channel gain.

When the conventional UFR technique is employed, the cell-centre area and cell-edge area are not distinguished by partitioning the total available frequency band and hence the received signal model may be described by Eq (1). However, when the FFR technique is employed, the signal received in the cell-centre area may be described by Eq (1) without any modification, while the signal received in the cell-edge area also obeys by Eq (1), provided that the IBI term is removed.

\section{System Throughput}

Let us now demonstrate how these SINR expressions may be mapped to the ultimate system performance metric formulated in terms of the achievable throughput $\eta$. The throughput may be characterized by the maximum successfully trans- 
mitted information rate, which is referred to as the system's goodput, given by:

$$
\eta(\gamma)=R_{M} R_{C}\left[1-P_{b l}(b, \gamma)\right],
$$

where $\gamma$ may represent either $\gamma_{c}$ of $\mathrm{Eq}$ (2) or $\gamma_{e}$ of $\mathrm{Eq}$ (4), $R_{M}$ denotes the 'rate' i.e. the throughput of the modulation scheme, while $R_{C}$ is that of the channel code. Still referring to $\mathrm{Eq}(5), P_{b l}(b, \gamma)$ represents the BLock Error Ratio (BLER) corresponding to the particular Modulation and Coding Scheme (MCS) employed. In this paper, we assume that Bit Interleaved Coded Modulation (BICM) [7] is employed, which relies on Gray mapped M-ary Quadrature Amplitude Modulation (QAM) [8], where we have $M=$ $2^{b}$ and $b=2,4,6$ represents the number of bits per QAM symbol. Hence the modem's throughput is $R_{M}=$ $b$. In our paper, we employ $2^{b}$-ary QAM combined with Rate Compatible Punctured Codes (RCPC) [9] having six selected MCSs, namely Mode $[1, \ldots, 6]$ of $\left[R_{M}, R_{C}\right]=$ $[(2,1 / 2),(2,3 / 4),(4,1 / 2),(4,3 / 4),(6,2 / 3),(6,6 / 7)]$.

In practice, obtaining the BLER curves for all the MCSs by simulation is time-consuming, especially in our multicell system-level evaluations. Let us hence circumvent this timeconsuming process with the aid of a more efficient semianalytical process. We commence by briefly highlighting the derivation of the BLER $P_{b l}(b, \gamma)$ of a $2^{b}$-ary QAM aided BICM scheme based on the BLER $P_{b l}\left(b=1, \gamma_{0}\right)$ of a BPSK modulated BICM scheme transmitting over an AWGN channel, where only $P_{b l}\left(b=1, \gamma_{0}\right)$ is evaluated by simulation. To this effect, we first define the appropriate base-line SINR $\gamma_{0}$ as that of a binary BPSK scheme, at which we have the same BLER as an arbitrary $b$-bit/symbol modem, which would typically require an SINR of $\gamma>\gamma_{0}$, i.e. where we have $P_{b l}(b, \gamma)=P_{b l}\left(b=1, \gamma_{0}\right)$. We note that the above-mentioned BLER equality holds only, when the mutual information per bit output by the demodulator obeys the relation of $I(b, \gamma)=$ $I\left(b=1, \gamma_{0}\right)$. This is because $I$ serves as the input of the channel decoder, and as long as $I$ remains the same, the ultimate BLER also remains the same. Hence, the mutual information per bit $I$ may be expressed as [7],

$$
\begin{aligned}
I(b, \gamma) & =1-\frac{2^{-b}}{b} \sum_{i=1}^{b} \mathbb{E}_{w} \times \\
& \left\{\ln \left[1+\sum_{j=1, j \neq i}^{b} \exp \left(-\frac{\left|u_{j}-u_{i}+w\right|^{2}-|w|^{2}}{1 / \gamma}\right)\right]\right\},
\end{aligned}
$$

where $w \sim \mathcal{C} \mathcal{N}(0,1 / 2 \gamma)$ is the complex-valued zero-mean Gaussian noise process and $u \in \mathcal{A}$ represents the constellation points in the QAM alphabet, with the $i$ th bit $\mathcal{A}$ being represented by $u_{i}, i \in[1, b]$. When fixing the mutual information per bit as $I(b, \gamma)=I\left(b=1, \gamma_{0}\right)$, we may try and find the baseline SINR $\gamma_{0}$ for $b=1$, where this BPSK modulated BICM scheme achieves the same per-bit mutual information(MI) $I$ as a higher-throughput $2^{b}$-ary QAM-aided BICM scheme. More explicitly, since the MI per pit $I\left(b=1, \gamma_{0}\right)$ is a monotonically increasing function of $\gamma_{0}$, its unique inverse exists. Hence this base-line SINR $\gamma_{0}$ may be expressed as the inverse-function
$\gamma_{0}=I^{-1}[b=1, I(b, \gamma)]$. This reveals the relation between $\gamma$ and $\gamma_{0}$, hence allowing us to find the BLER $P_{b l}(b, \gamma)$ of a $2^{b}$-ary QAM-aided BICM scheme from that of the simulated BLER of a BPSK modulated BICM scheme, i.e. from $P_{b l}(b=$ $\left.1, \gamma_{0}\right)$, which may be formulated as:

$$
P_{b l}(b, \gamma)=P_{b l}\left\{b=1, I^{-1}[b=1, I(b, \gamma)]\right\} .
$$

As a result, the BLER $P_{b l}(b, \gamma)$ of the above MCSs may be obtained with the aid of $\operatorname{Eq}(7)$, where $P_{b l}\left(b=1, \gamma_{0}\right)$ is obtained from the Monte-Carlo simulation of a BPSK modulated BICM scheme employing the above-mentioned RCPC having constituent code-rates of $R_{C}=[1 / 2,3 / 4,2 / 3,6 / 7]$ in an AWGN channel. Finally, the associated effective throughput $\eta(\gamma)$ of Eq (5) expressed in terms of 'bits/s/Hz' may be obtained by assuming a Nyquist roll-off factor of zero. Owing to space limitations, the detailed link-level simulation results are not explicitly shown here.

\section{Performance Evaluation}

\section{A. Simulation Assumptions}

In our simulations, we consider the Urban-Macro propagation scenario of [4], where the BS-to-BS distance is $D=3 \mathrm{~km}$ and the pathloss expressed in $\mathrm{dB}$ is $34.5+35 \log _{10}\left(d_{0}\right)$, with $d_{0}$ being the distance between any transmitter and receiver in meters. We assume furthermore that the total transmitter power is $P=46 \mathrm{dBm}$ and the noise power at the MS is $-174 \mathrm{dBm} / \mathrm{Hz}$, when an operating bandwidth of $10 \mathrm{MHz}$ is considered [4]. Moreover, the optical fibre link's normalised Signal-to-Noise-Ratio (SNR) is $20 \mathrm{~dB}$ and the length of the optical fibre is $L_{R i}=0.68 R, \forall i$.

The cell-centre area's radius $r$ may be defined as the maximum radius, where the throughput of $\eta=2 b i t s / s / H z$ may be maintained in a conventional cellular system employing the classic UFR technique, but beyond which it drops below $\eta=$ 2bits/s/Hz. When employing DASs in a FFR-based cellular system, the transmit power of the BS should be sufficiently high to ensure that the average throughput maintained at a distance of $r$ remains exactly as high as that of the classic UFR technique, while the rest of the power is evenly allocated to the RAs. Hence, the resultant power sharing regime of all the transmitters obeys $P_{B}=2 P / 5$, while we have $P_{R}=P / 10$ and $P_{R}=P / 20$, when $K=6$ and $K=12$ RAs are considered, respectively. Finally, the ultimate throughput is obtained by averaging the SINR over 30000 simulation runs and then substituting it into Eq (5) and Eq (7) .

\section{B. Comparison of the SINR}

Fig. 2 characterizes the SINR as a result of the IRI imposed by the $K=6$ RAs of the FFR aided system, where the SINR at any point $Z$ along the entire radius $R$ seen at the top-left conner of Fig. 2 was compared in four scenarios, as discussed below. First, the best-and-worst-case directions $\theta_{\text {best }}=0$ and $\theta_{\text {worst }}=\pi / 6$ were considered, which represented the direct BS to RA direction and the angle halfway between two adjacent RAs. In the absence of IPI, the SINR difference between the best and worst scenarios is the highest in the middle position i.e. at angle $\theta_{\text {best }}$ and $\theta_{\text {worst }}$, where the 


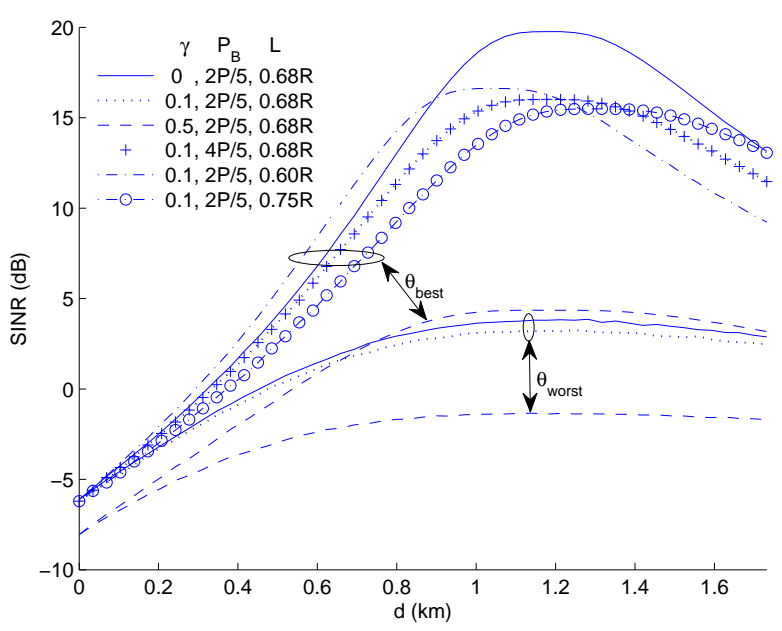

Fig. 2. SINR comparison of the $K=6$-aided FFR system for four different pairs of $\theta_{\text {best }}$ vs $\theta_{\text {worst }}$ and for different amount of IPI associated with $\beta=0,0.1,0.5$ and for $P_{B}=4 P / 5,2 P / 5$ and for different distances between the BS and RA connected by optical fibre, namely for $L_{R i}=$ $0.6 R, 0.68 R, 0.75 R$.

interference imposed by the adjacent RAs is approximately as strong as the desired signal. Secondly, when the IPI control parameter $\beta$ of the fibre is increased from 0 to 0.5 , the SINR experienced in the direction $\theta_{\text {best }}$ is more seriously reduced than in the direction of $\theta_{\text {worst }}$ owing to the same halfway angular position. Hence, the achievable performance in the direction $\theta_{\text {best }}$ is sensitive to the imperfections imposed by the optical fibre. Thirdly, having a higher distance between the $\mathrm{BS}$ and the RAs corresponds to having a longer optical fibre, which introduces a higher IPI, although naturally, provides the MSs roaming near the cell-boundary with an increased SINR. Hence we may expect that there exists an optimum position for the RAs connected by lossy optical fibre. Finally, two different power allocation strategies associated with $P_{B}=$ $4 P / 5, P_{R}=P / 30$ and $P_{B}=2 P / 5, P_{R}=P / 10$ are used, where the corresponding two curves nearly coincide with each other in Fig. 2, which illustrate that $P_{R}=P / 30$ and $P_{R}=P / 10$ achieve a similar SINR performance. Hence, there is no need to dissipate as high a total power as $P$, if we have $P_{B}=2 P / 5, P_{R}=P / 30$, because $P_{B}+6 P_{R}=3 P / 5$, which indicates that as much as $2 P / 5$ power is saved, when compared to the traditional UFR and FFR systems. Hence our design has the potential to qualify as a 'green' regime by saving $40 \%$ power dissipation.

\section{Comparison of the Throughput in the Best Direction}

Fig. 3 compares the throughput of the classic UFR system, of the traditional FFR system and of the RA-aided FFR assisted systems using $K=6$ and $K=12$ along the best direction, when suffering from different levels of IPI in the optical fibre. It can be seen in Fig. 3 that upon assigning a transmit power of $P_{B}=2 P / 5$ in the cell-centre area, the throughput of the RA-aided FFR-assisted system is almost the same as that of the traditional UFR and FFR systems, when these two systems assign the full transmit power to the BS. By
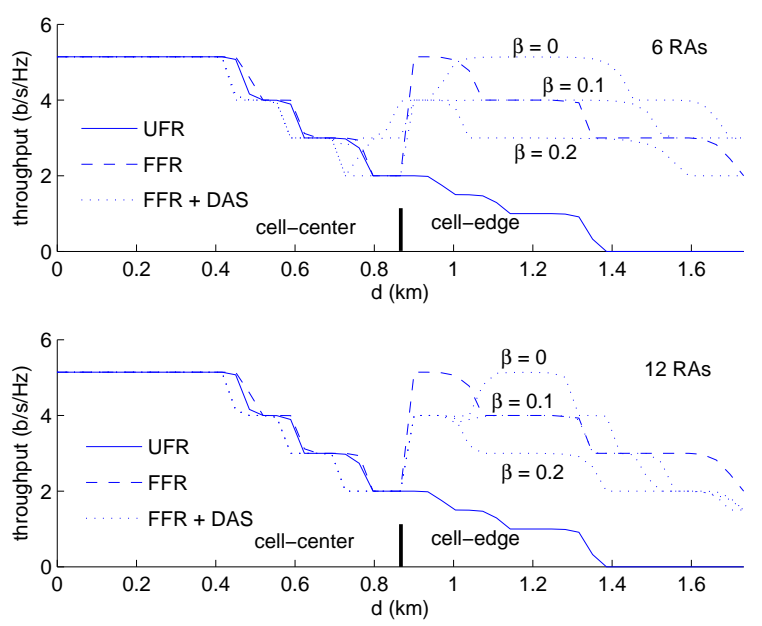

Fig. 3. Throughput comparison of the traditional UFR system, FFR system and the $K=6$ and $K=12$ RA-aided FFR-assisted systems along the best direction when having different amounts of IPI imposed by the optical fibre.

contrast, when the cell-edge area is considered, the throughput of the conventional UFR scheme becomes lower than $\eta=2$ bits/s/Hz, as illustrated by the solid line of Fig. 3. When a RAaided FFR-assisted system is employed in conjunction with either $K=6$ or $K=12 \mathrm{RAs}$, a throughput of $\eta>2 \mathrm{bits} / \mathrm{s} / \mathrm{Hz}$ is achieved, even in the presence of strong IPI associated with $\beta=0.5$. However, Fig. 3 also demonstrates that the employment of DASs combined with the FFR technique does not necessarily lead to combined throughput enhancements in the presence of IPI. This may be verified by observing in Fig. 3 that the cell-edge throughput is reduced compared to the classic FFR system for both the $K=6$ or $K=$ 12 configurations, when the IPI control parameter becomes $\beta \geq 0.2$. As long as the IPI can be neglected, the RA-aided FFR assisted system achieves a consistently higher cell-edge throughput than the FFR system. Overall, this phenomenon makes the combination of these two techniques less attractive. In addition, when comparing the throughpout curves of both the $K=6$ and of the $K=12$ configurations, Fig. 3 implies that different DAS configurations have different throughput distributions across the coverage area, which may be better explained by observing Fig. 5 .

\section{Comparison of the Entire Cell Throughput}

Fig. 3 only characterized the achievable throughput in a given direction. In order to compare the attainable throughput across the entire cell, we calculated the corresponding percentage of the entire cell over which a certain throughput $\eta$ was maintained, as illustrated in Fig. 4. It can be seen that employing $K=6$ RAs achieves a higher throughput than $K=12$ RAs for both the ideal and realistic imperfect optical fibre scenarios. However, we emphasise that this does not necessarily lead to the conclusion that the $K=6$ RA-aided configuration is better than the $K=12$ RA configuration, as confirmed by observing the throughput contours in the absence of IPI in Fig. 5. More explicity, the cell-centre area's 

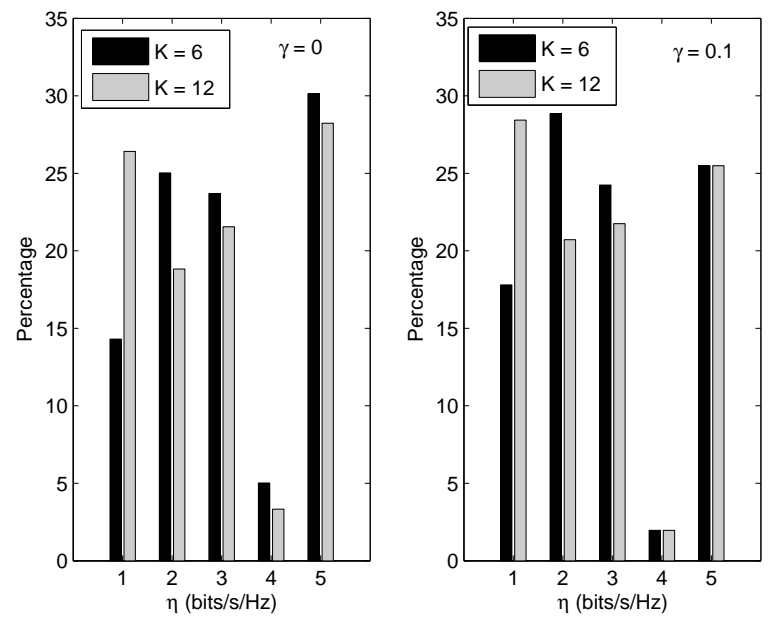

Fig. 4. The percentage of the cell throughput for both $K=6$ and $K=12$ RAs aided FFR enabled system with and without IPI.

throughput contours recorded in Fig. 5 for both configurations are similar. However, the throughput distribution of the $K=6$ RA-aided configuration recorded for the cell-edge area tends to be more 'patchy', while that of the $K=12$ RA-assisted configurations tends to cover the entire outer ring more continuously. As a result, the general conclusion emerging for the comparison of these two configurations is that the optimum positioning of DASs should take into account the expected user-distribution and user-load.

\section{CONCLUSIONS}

The achievable throughput across the entire cellular area of a RoF aided DAS relying on FFR and a realistic optical fibre backhaul was investigated. Our simulation results demonstrated that the throughput distribution of the $K=6$ RA was patchy, while that of the $K=12$ RA-aided FFR had a more uniform character. Furthermore, the tradeoffs between using DAS-aided FFR and tradition FFR systems depend on the impairments introduced by the lossy optical fibre, where the fibre-induced imperfections were largely neglected in most of the existing studies. When the IPI is low i.e. we have $\beta \leq 0.1$, the DAS aided FFR beneficially enhances the celledge area's throughput without requiring any extra transmit power in comparison to the traditional transmit strategy. On the other hand, in the presence of strong IPI the benefits of RoF aided DASs may erode.

\section{REFERENCES}

[1] Y. Xiang and J. Luo, "Inter-cell interference mitigation through flexible resource reuse in OFDMA based communication networks," Proceedings of European Wireless, vol. 43, pp. 1-7, Apr. 2007.

[2] C. Wan and J. Andrews, "Downlink performance and capacity of distributed antenna systems in a multicell environment," IEEE Transactions on Wireless Communications, vol. 6, pp. 69- 73, Jan. 2007.

[3] D. Wake, M. Webster, G. Wimpenny, and et al, "Radio over fiber for mobile communications," in IEEE International Topical Meeting on Microwave Photonic, Oct. 2004, pp. 157-160.

[4] "Spatial channel model for MIMO simulations," Technical specification group radio access network, 2008, ftp://ftp.3gpp.org/.
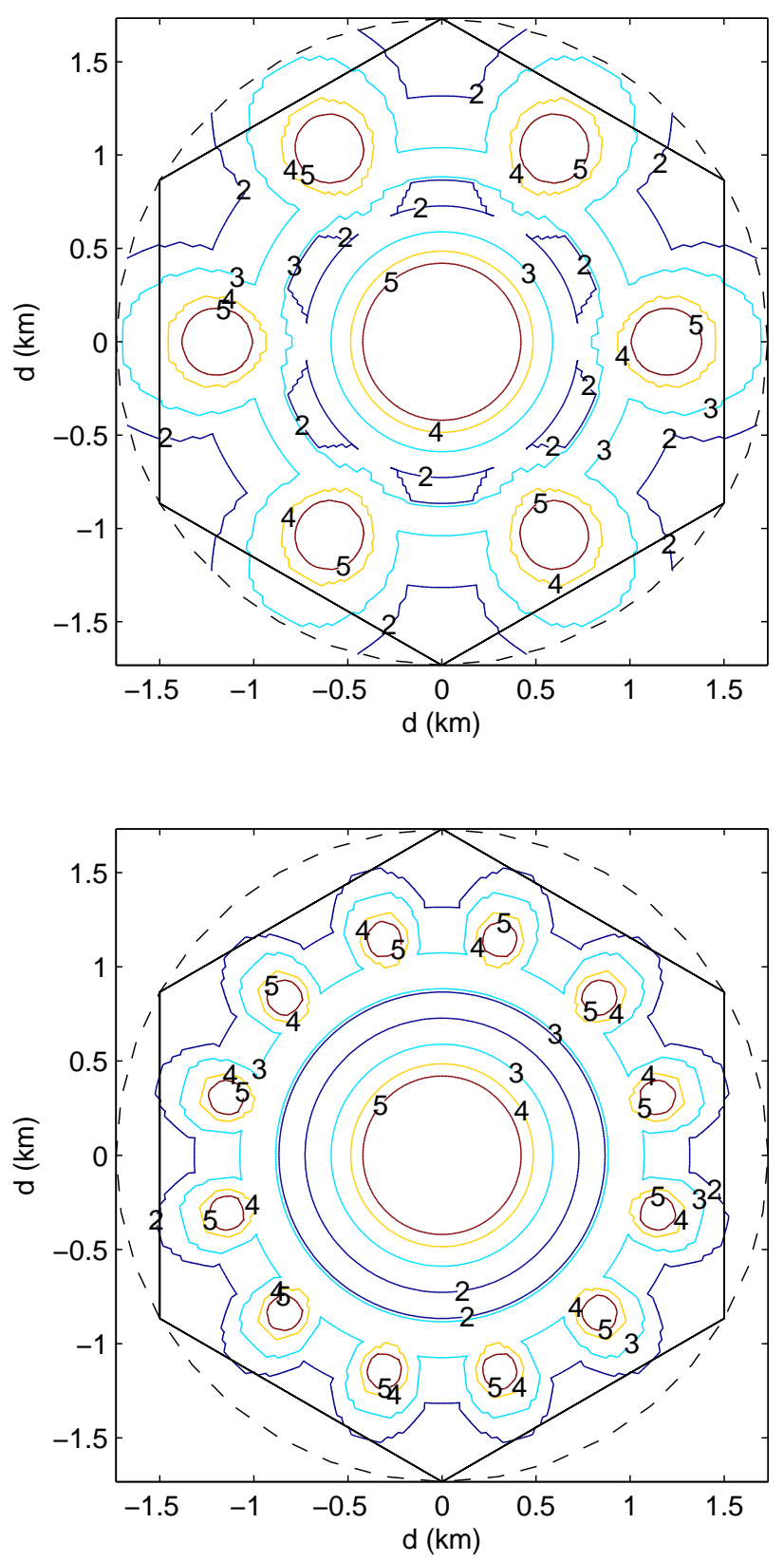

Fig. 5. Throughput contours for the $K=6$ and $K=12$ RA-aided FFRassisted systems, when assuming the absence of IPI in the optical fibre.

[5] G. P. Agrawal, Fiber Optical Communications Systems, Third, Ed. John Wiley \& Sons, Inc., 2002.

[6] J. Shaw, Mathematical principles of optical fiber communications. Philadelphia: Society for Industrial and Applied Mathematics, 2004.

[7] G. Caire, G. Taricco, and E. Biglieri, "Bit-interleaved coded modulation," IEEE Transactions on Information Theory, vol. 44, pp. 927-946, May 1998.

[8] L. Hanzo, S. X. Ng, T. Keller, and W. T. Webb, Quadrature Amplitude Modulation: From Basics to Adaptive Trellis-Coded, Turbo-Equalised and Space-Time Coded OFDM, CDMA and MC-CDMA Systems. Wiley-IEEE Press, 2004.

[9] J. Hagenauer, "Rate-compatible punctured convolutional codes (RCPC codes) and their applications," IEEE Transactions on Communications, vol. 36, pp. 389-400, Apr. 1988. 